\title{
Wide Optical Lenses for Atomic Beams
}

\author{
Ole Steuernagel \\ School of Physics, Astronomy, and Mathematics, University of Hertfordshire, Hatfield, AL10 9AB, UK
}

The field of atom-optics offers considerable potential in fundamental and applied physics, both for imaging atoms and their deposition (to create nano-structures) [1]. Since Ashkin's group's work in 1978 [2], many techniques to focus or concentrate atomic beams have been tried: transmission gratings, holographic reflectiongratings, electro-static lenses, magnetic lenses or mirrors, nano-apertures and optical setups, relying on the dipole force [1]. These approaches suffer from various shortcomings. A common problem is the lack of wide lenses with large numerical apertures.

The superpositions of several odd modes of the well known Hermite-Gaussian TEM $_{m n}$-modes, $\psi_{m, n}$, can help in this regard [3]. In a configuration, where an atomic beam is to be focussed by crossing through a laser beam one can generate [4] a wide cylindrical atom-lens (two such crossed lenses can constitute a parabolic lens) using an electric field composed of a suitable combination $E_{2 J+1}(\mathbf{r}) \propto \sum_{j=0}^{J} c_{2 j+1} \psi_{2 j+1,0}(\mathbf{r})$ of odd modes (first index ' $2 j+1$ ' refers to $x$, second index ' 0 ' to $y$ direction - along which the atomic beam travels). Here we work in the paraxial approximation throughout and can therefore assume that the scalar modes $\psi_{2 j+1,0}(\mathbf{r})$ represent (e.g., linearly polarized) electro-magnetic laser-field modes.
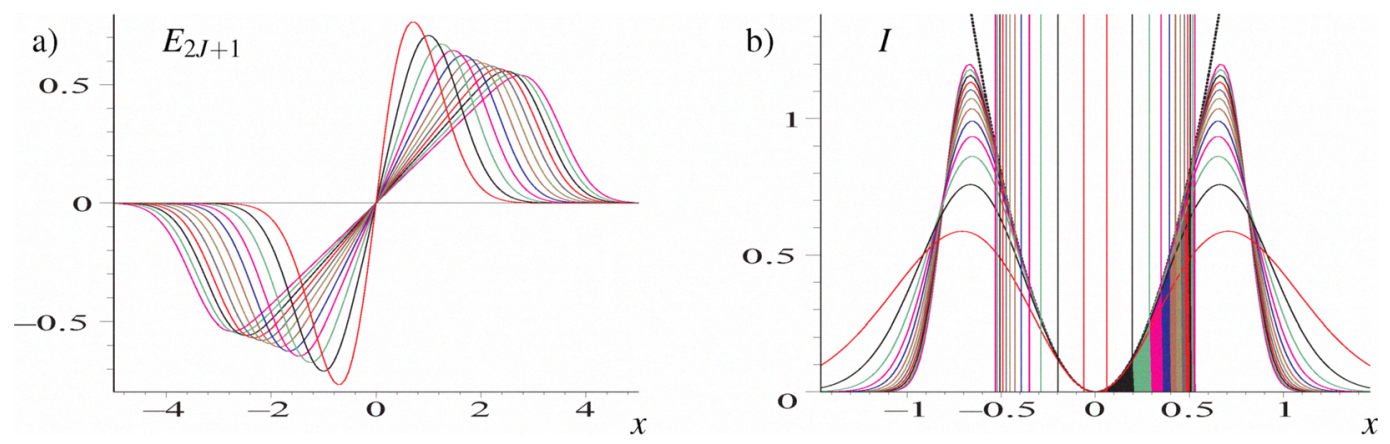

Fig. 1 a) Superpositions of intensity-normalized beams comprising up to $23^{\text {rd }}$ order modes (' $2 J+1$ ' $=23$; in units of beam waist $w_{0}$ ). b) Intensity profiles of beams comprising up to $23^{\text {rd }}$ order modes and their $1 \%$ deviation marks (same units).

The effectively linear dependence, on the $x$-direction, of the (y-integrated) electric field profile, see Fig. 1 a) yields, compared to single mode fields, greatly extended parabolic intensity profiles, see Fig. 1 b). This allows for wide lenses with large numerical apertures and considerable laser power savings.

The vertical bars in Fig. 1 b) delineate the areas where each intensity curve deviates from the enveloping ideal parabola (dotted line) by one percent and maps out the useful area of the lens.

Note that the (highlighted) areas, in Fig. 1 b), represent the relative beam power contributing to the formation of the useful parts of the atom-lenses in each case. Clearly very considerable laser-power savings are achievable with this method [3].

A crossed combination of superposition-beams allows the creation of a wide parabolic spherical lens for which Gouy-dephasing of the modes is not a problem [3].

For atomic lens design, based on the optical dipole force, it is necessary to coherently superpose suitable laser modes in order to create wide thin parabolic lenses with large numerical apertures. Only in this manner can the tiny diffraction limits promised by the extraordinary smallness of the de Broglie wavelengths of atomic beams be realized.

\section{References}

[1] D. Meschede and H. Metcalf, "Atomic nanofabrication: atomic deposition and lithography by laser and magnetic forces", J. Phys. D: Appl. Phys. 36, R17 (2003).

[2] J. E. Bjorkholm, R. R. Freeman, A. Ashkin, and D. B. Pearson, "Observation of focussing of neutral atoms by the dipole forces of resonance-radiation pressure", Phys. Rev. Lett. 41, 1361 (1978).

[3] O. Steuernagel, "Optical lenses for atomic beams", Phys. Rev. A 79, 013421 (2009).

[4] O. Steuernagel, E. Yao, K. O'Holleran, and M. Padgett, "Observation of Gouy-phase-induced transversal intensity changes in focused beams", J. Mod. Opt. 52, 2713 (2005).

[5] G. M. Gallatin and P. L. Gould, "Laser focussing of atomic beams", J. Opt. Soc. Am. B 8, 502 (1991). 